\title{
A multi-sensor system for enhancing situational awareness and stress management for people with ASD in the workplace and in everyday life
}

\author{
Filippo Sanfilippo \\ Dept. of Science and Industry Systems \\ University of Southeast Norway (USN) \\ filippo.sanfilippo@usn.no
}

\begin{abstract}
Autism spectrum disorders (ASD) present challenges for affected people at work and in everyday life. The barrier increases further with changing environmental situations. Deviations in factors like lighting or sound may lead to increased stress. The intervention plans to instil positive behaviour support (PBS) suggest that a customised environment can minimise the impacts due to these variations. This work proposes a novel framework which leverages the information from multi-sensor channels in a combined manner to customise the environment so that situational awareness (SA) can be improved. The proposed framework allows for monitoring the environment by combining the information from different sensor channels including both personal sensors (i.e. on board of a mobile device) as well as environmental sensors/actuators (i.e. embedded in smart-buildings). In this preliminary work, the system architecture is introduced. To demonstrate the potential of the proposed system, a case study is also considered through the development of a prototype for a mobile application and by reporting results on a scale model of a smart workplace with customisable environment.
\end{abstract}

\section{Introduction}

The Autism spectrum disorder (ASD) is a developmental disorder that affects communication and behavior. The autism spectrum is wide and includes different diagnoses that are related to each other [1], such as child autism (infantile autism), Asperger's syndrome, atypical autism and unspecified pervasive developmental disorder (PDD-NOS). Many subjects within the spectrum also have additional diagnoses and difficulties of differing and partly comprehensive characters. Examples of this variety may be developmental disabilities of different degrees, severe language and communication difficulties (some remain language-free), epilepsy, mental disorders,

\author{
Kiran B.Raja \\ Dept. of Science and Industry Systems \\ University of Southeast Norway (USN) \\ filippo.sanfilippo@usn.no
}

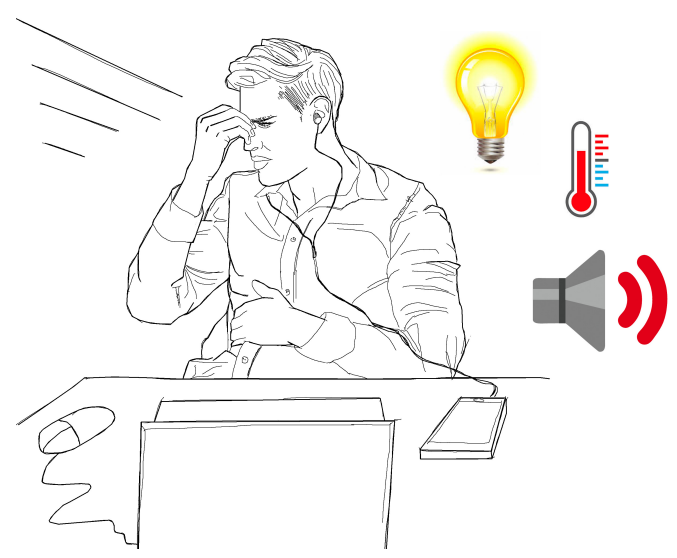

Figure 1. The underlying idea of designing a multi-sensor system for enhancing situational awareness (SA) and stress management for people with ASD in the workplace and in everyday life.

various somatic disorders and extensive sleep problems, all of which impact the productivity at work [2]. ASD is further considered as a pervasive disorder that can cause sensory-perceptual anomalies, such as a hypersensitivity to contact with other people [3]. A challenge is therefore to continuously and non-intrusively monitor the change in the subjects expression to adapt and minimise the impact of sensory-perceptual anomalies. This is even more challenging when considering the workplace environment which houses number of people, sensors and a variety of office landscapes.

There are some common characteristics within ASD, especially when it comes to deviations in the ability of social interaction, limited/narrow interests, little repertoire of activities and monotonous way of doing things. Nevertheless, the "main challenge" is the large variation of ASD due to enormous individual differences and complexities of each individual. It is widely accepted that the heterogeneity in the functional level within ASD is greater than in other clinical groups, and that the identification of severity and additional difficulties is crucial to obtain personalised diagnoses. Addressing the challenges is therefore 
demanding and requires cutting edge expertise and collaboration between experts, local people and local authorities.

In recent years, a sharp increase in the number of subjects with autism spectrum diagnoses has been registered. It is estimated that about $1 \%$ of the population may be within the autism spectrum worldwide [2]. Considering this population, there is a considerable internal variation and distribution, which includes a large number of children, adolescents, adults and elderly, who need lifelong but different and individualised levels of help and follow-up throughout their lives.

At the same time, the employment of people with ASD has been reported with remarks on the extraordinary ability to focus on a specific task over a period of time. From the industry perspective, increased efforts are being considered to mitigated stress at the workplace for people with ASD or similar challenges. For instance, according to initial findings at Microsoft, providing feedback more often, sharing recaps of meetings in writing, and giving clear expectations have helped employees with ASD to successfully increase situational awareness (SA) ${ }^{1}$. At the heart of Microsoft findings is a larger focus on changing the unemployment and underemployment rate for people with ASD and similar challenges, which is currently $80+\%$ [4]. It's therefore imperative to welcome and successfully include these subjects into the workplace. As there are many good reasons to include individuals with ASD into the regular workforce, other companies are also converging, i.e. SAP, JP Morgan Chase, EY, Ford Motor Company, and DXC technologies. In this perspective, it is important to cater the needs of people with ASD and similar challenges so that enabling tools and technology can be provided for their special assistance.

Although, the inclusion of people with ASD is one end of the problem, the greater problem is in understanding the exact needs of the people with ASD at workplaces to make it welcoming. A stronger hypothesis and the results from earlier works has suggested the hypersensitivity of people with ASD as a result of sensory-perceptual anomalies [3]. However, the remedial measures to mitigate the sensory-perceptual anomalies are still missing. The need for structured and scientific understanding of customisable solutions for ASD is still to be fulfilled.

The main contribution of this work is in designing and prototyping a multi-sensor system for enhancing

\footnotetext{
${ }^{1}$ https: //blogs.msdn.microsoft. com/ accessibility/2018/04/02/autismawareness2018/
}

SA and stress management for people with ASD in everyday life and especially in the workplace, as shown in Fig. 1. Such a system makes it possible to monitor and combine information from different sensor channels, such as personal sensors (i.e. on board of a mobile device) or environmental sensors (i.e. embedded in smart-buildings), with the aim of increasing SA and to minimise the impact of stress. In this preliminary work, the system architecture is presented. Related initial experiments are carried out to validate the efficiency of the proposed technology. In particular, the developed prototype of a mobile application is tested on a scaled model of smart workplace. The development of the presented system is supported by the Autismeforeningen i Norge (AN), which is the autism association in Norway. This association was founded in 1965 and has approx. 5,000 members. The association is an organisation of people with diagnoses within the autism spectrum, parents and other relatives, professionals and other interested parties.

The paper is organised as follows. A review of the related research work is described in Section 2. The proposed multi-sensor system for enhancing SA and stress management for people with ASD in the workplace and in everyday life is presented in Section 3. As a case study, the developed prototype of a mobile application is tested on a scale model smart workplace in Section 4. In Section 5, related experimental results are presented. Finally, conclusions and future work are outlined in Section 6.

\section{Related research works}

ASD has been reported to cause sensory and perceptual anomalies including hypersensitivity for surroundings and contact with other within the environment [3]. A set of earlier works have indicated the use of music assisted therapy to relieve the agitated behaviour from people with ASD [5]. A set of children with autism were reported to have success with effective use of music therapy in number of sessions. The survey on the use of music therapy also indicated that $56 \%$ of child psychiatrists and $14.5 \%$ of pediatricians preferred it [6]. While this indicated the proportion for the children, there are no existing systematic studies for the adults on the impact of music in employment setting. The sensory processing issues were further reported in $85 \%$ of study participants who were affected with ASD. The key observation was that the abnormal sensory processing was reported by people diagnosed with autism. Another work observed the insensitivity involving all of the sensory modalities (tactile, oral, auditory, vestibular, and visual systems) for people with 


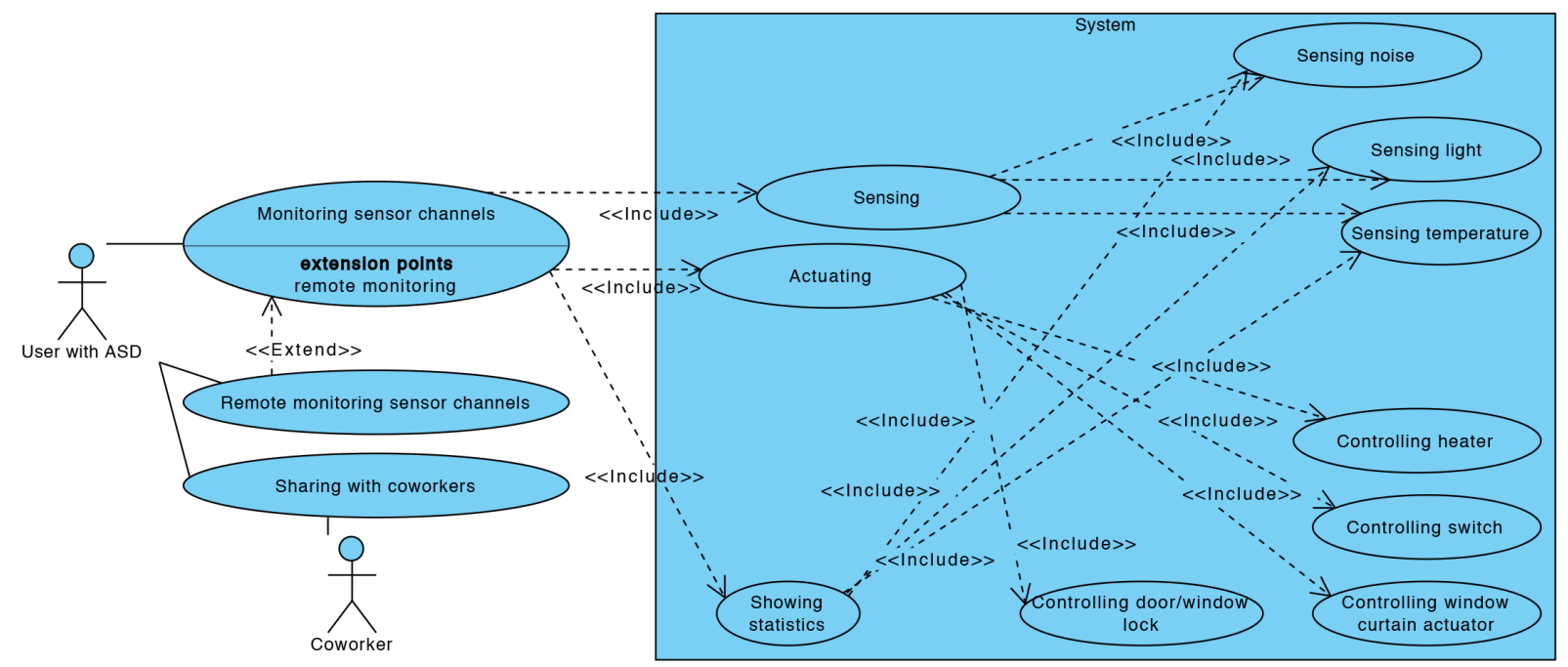

Figure 2. The considered use case diagram.

ASD [7]. A study in these direction also reported an inductive, qualitative thematic analysis with four main themes encompassing both positive and negative sensory experiences [8]. The key outcome suggested the importance of particular aspects of stimuli in their perception to instill the control over stimuli. The work also indicated how emotions/mental states could be impacted by sensory stimuli. Thus, the studies encourage the need for further experiments on the personalised work-space to make it freiendly for people with ASD.

In another parallel set of research works, the preliminary studies have indicated the ability of the people with ASD to focus on specific tasks ${ }^{23}$. However, the impact on the sensory modalities of people with ASD is reported to impact the productivity and the focus [3]. The need is therefore to gauge the sensory responses and to adapt the environment at the workplace through proactive measures. This need can be supported by the current technology trends related to smart-buildings. Within a smart-building many sensors and actuators are interconnected to form a control system. Nowadays, the deployment of a building control system is complicated because of different communication standards. To tackle this challenge, different researchers are designing novel interfaces to facilitate sensor integration for smart-buildings. For instance, a web services-based approach to integrate resource constrained sensor and actuator nodes into IP-based networks was presented in [9]. This research areas is also in line with a novel holistic view of how sensing technologies can play a

\footnotetext{
${ }^{2}$ https: / / sverigesradio.se/sida/artikel.aspx? programid=493\&artikel=6371833

3 https: / / www.nimh.nih.gov/health/topics/ autism-spectrum-disorders-asd/index.shtml
}

crucial role in smart cities. A range of applications and technical challenges associated with these applications are discussed in [10]. However, to the best of the authors knowledge, there are no works that have currently reported the use of personal and environmental sensors to work cooperatively so that conducive environments at the work-place can be achieved. These background works have motivated us to create a new framework for making the workplace friendly to people with ASD.

\section{System Architecture and mobile application}

This section presents the system architecture proposed in this work. The Unified Modeling Language (UML) [11] is adopted to present the system architecture and to better highlight the main features of the proposed system. Note that the UML is used hereafter to present blueprints (the real implementation of the system may differ).

A UML use case diagram is shown in Fig. 2 to describe the set of processes (use cases) that a user should or could perform with the proposed framework. In particular, a user with ASD or similar challenges can monitor different sensor channels to cope with changing environmental situations. This monitoring process includes the possibility for the user to sense and collect information from different sensors, including both personal sensors (i.e. on board of a mobile device) as well as environmental sensors/actuators (i.e. embedded in smart-buildings). In this way, the system allows the user for sensing different changing environmental sources, such as noise, light and temperature. The monitoring process also includes the possibility of controlling different devices 


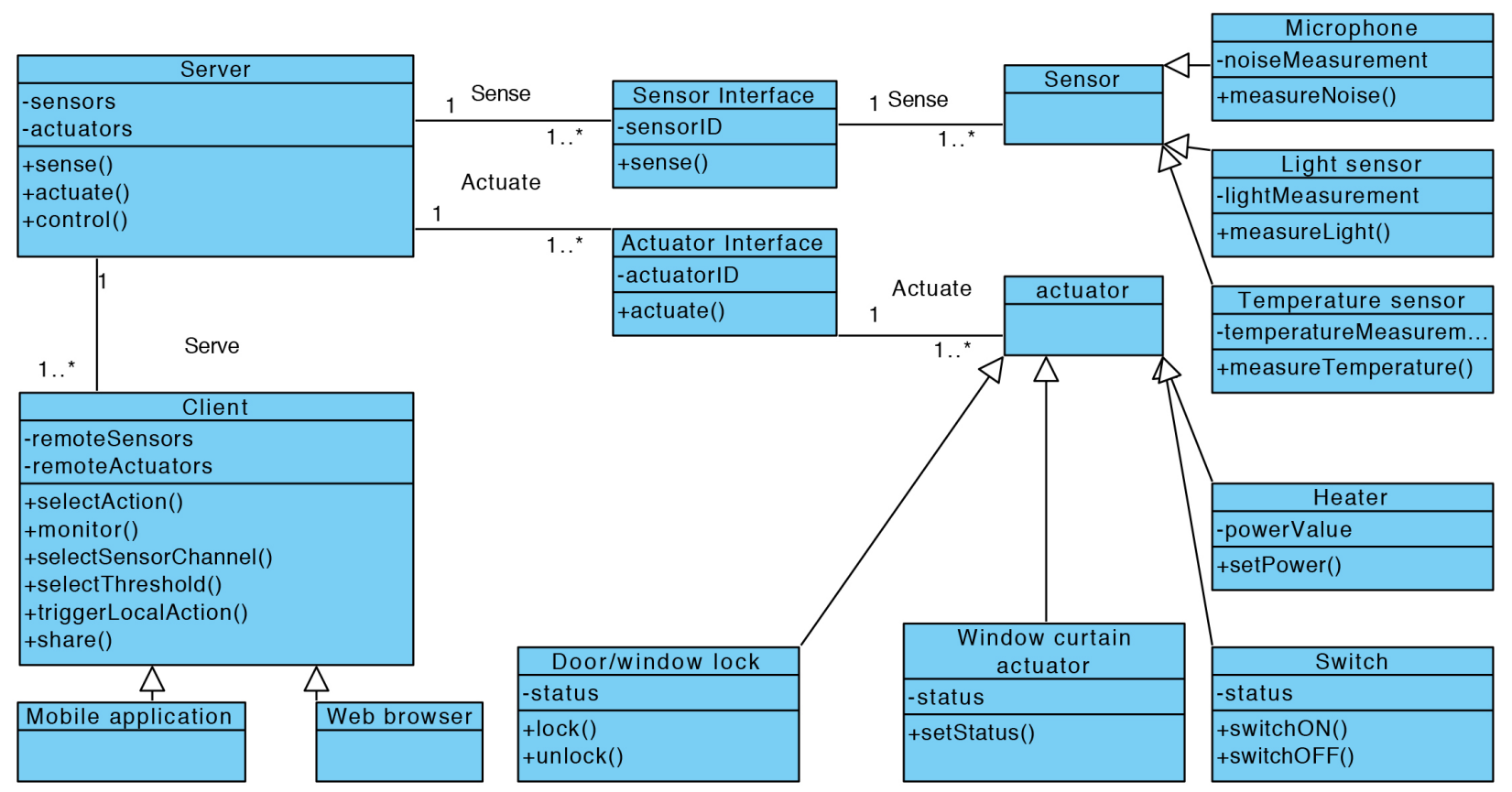

Figure 3. The considered class diagram.

embedded in the workplace, such as heaters, light switches, window curtains and door locks. Further, the possibility of collecting data and showing statistics to the user is considered. Extension of the main use case are the possibility of remotely accessing the system and the opportunity for sharing the monitoring of specific channels with coworkers/family/friends circles. This possibility allows for increasing both personal as well as collective $\mathrm{SA}$.

A UML class diagram is shown in Fig. 3 to describe the structure of the proposed framework by showing the considered classes, their attributes, operations (or methods), and the relationships among objects. A client-server pattern is adopted. The server is provided with both a sensor (microphone, light sensor, temperature sensor) and actuator interfaces (heater, switch, window curtain actuator, door/window lock). Each sensor is provided with specific attributes and operations to measure and store data from different environmental channels (i.e. to monitor noise, light and temperature). Each actuator is provided with specific attributes and operations to actuate different appliances (i.e. doors, windows, switches, heaters). A mobile application operates as a client and remotely communicates with a server where the logic of the presented architecture is implemented. The client can also be implemented through a web browser.

\subsection{Server}

The server is designed as a multi-threading control program. Strict real-time criteria must be adopted. This allows to simultaneously and efficiently collect data from different sensors. The server is interfaced with different sensors and actuators through specific sensor and actuator interfaces. The server provides different operations, such as sense(), actuate() and control(). The serve is the core of the proposed framework. It makes it possible to develop alternative control method for both personal sensors (i.e. on board of a mobile device) as well as environmental sensors/actuators (i.e. embedded in smart-buildings). The framework does not impose any limitation regarding the internal implementation of these operations. However, each possible control method must be compliant with the provided interfaces.

\subsection{Mobile application (client)}

To take full advantage of the proposed system architecture, a mobile application is proposed. The mobile application works as a client and remotely communicates with the server. The user interface mockup is shown in Fig. 4.This mobile application enables the users to achieve the following tasks:

- Workplace/home monitoring for enhancing SA. By using different sensors embedded on the mobile device of the user (i.e. microphone, 


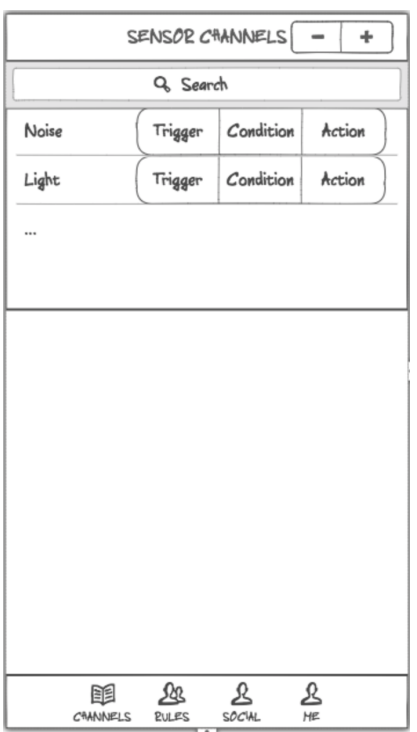

(a)

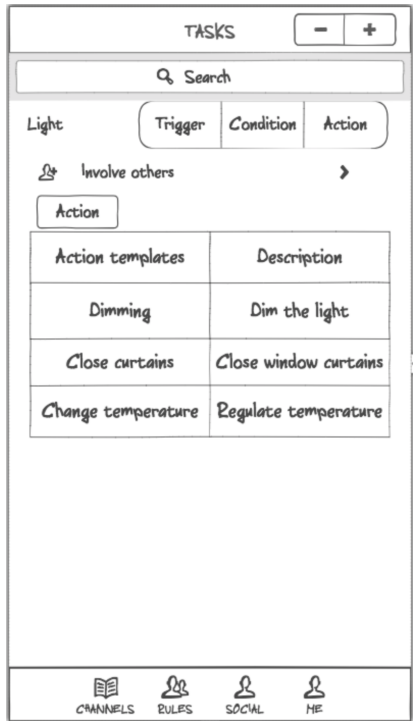

(b)

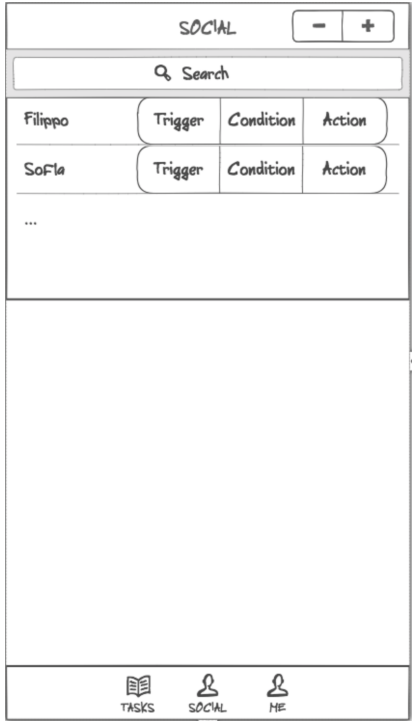

(c)

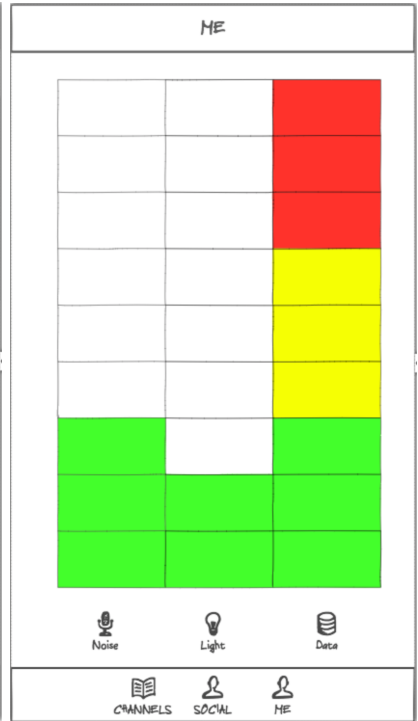

(d)

Figure 4. The proposed user interface for the mobile application: (a) the list of different sensor channels to be monitored; (b) the threshold and settings for a specific sensor channel; (c) the coworkers/family/friends circles;

(d) the real-time visualisation of different sensor channels.

camera, proximity sensor, ambient light sensor, accelerometer, gyroscope, compass, barometer) as well as smart sensors potentially embedded in the building, various information can be collected with a multi-sensor approach to constantly monitor the workplace/home environments. The list of sensor channels is shown in Fig. 4-a;

- Personalised sensor selection. The mobile application allows the user for personalising and selecting distinct environmental inputs to be monitored, according to specific needs. The user can also set certain thresholds for these inputs, according to individual wishes, shown in Fig. 4-b;

- Personalised situation handling and stress management. The mobile application allows the user for customising and selecting different actions or set of actions that will be triggered and executed when particular sensor thresholds are reached (i.e. a relaxing song will be played, a calming figure will be visualised). This may help the subject to better handle stress due to changing situations;

- Social involvement and communication improvement. When particular sensor thresholds are reached, personalised messages can be sent to different coworkers/family/friends circles, alerting them of the ongoing stressful situation and so that collective SA can be increased. The list of coworkers/family/friends circles is shown in Fig. 4-c. This may help the subjects to better handle communication while facing challenging situations;

- Intuitive visualisation of sensor data to improve SA. Different statistics can be intuitively visualised to increase SA as shown in Fig. 4-d.

\section{Conceptual framework case study}

The physical scale model smart workplace is shown in Fig. 5. The model includes four rooms with different embedded sensors and actuators, such as light-emitting diode (LED), photo sensors, thermostats, heaters and fans.

In this preliminary work, the continuous control and monitoring of a room's light intensity is considered. This is simulated by using the light sensor embedded on the user's mobile device to monitor and control the brightness of the LEDs embedded in the considered scale model smart workplace.

Different control algorithms can be adopted [12]. In this work, a Proportional-Integral-Derivative (PID) controller was designed. To tune the PID parameters, numerous methods exist, such as the one proposed in [13]. In this work, a trial-and-error tuning method has been adopted for the sake of simplicity.

For this first prototype, a mobile application is 


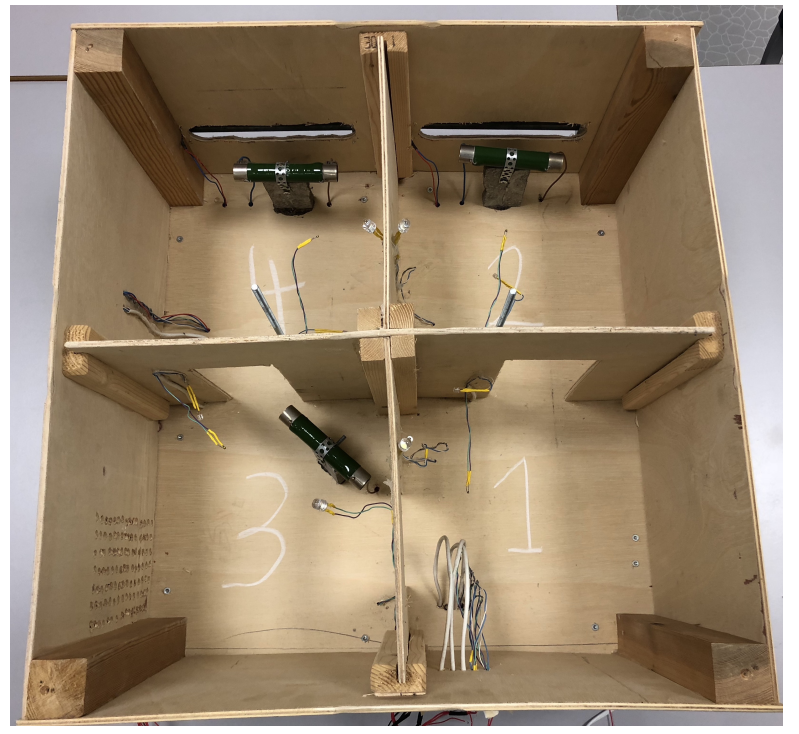

Figure 5. A scale model smart workplace was used to perform initial experiments and to validate the efficiency of the proposed technology.

developed using Android [14] as a platform. An Arduino micro controller board [15] is adopted to control the physical scale model smart workplace. The Bluetooth [16] protocol is used for the communication between the mobile application and the model.

Concerning the considered case study, a demo video is available on-line ${ }^{4}$.

\section{Experimental results}

Related simulation are carried out to test the proposed framework. In detail, the following scenario is considered:

- a desired luminosity threshold (set-point) is set by the user through the mobile application for a specific room of the physical scale model smart workplace;

- initially, the active control algorithm is disabled;

- successively, active control is applied to regulate the room luminosity according to the desired threshold.

For this particular scenario, a time plot is shown in Fig. 6. When no active monitoring and control is applied, deviations are measured. Contrary, the measure level of luminosity matches with the desired threshold when active monitoring and control is applied.

\footnotetext{
${ }^{4}$ https://youtu.be/sw4PiFulX-8
}

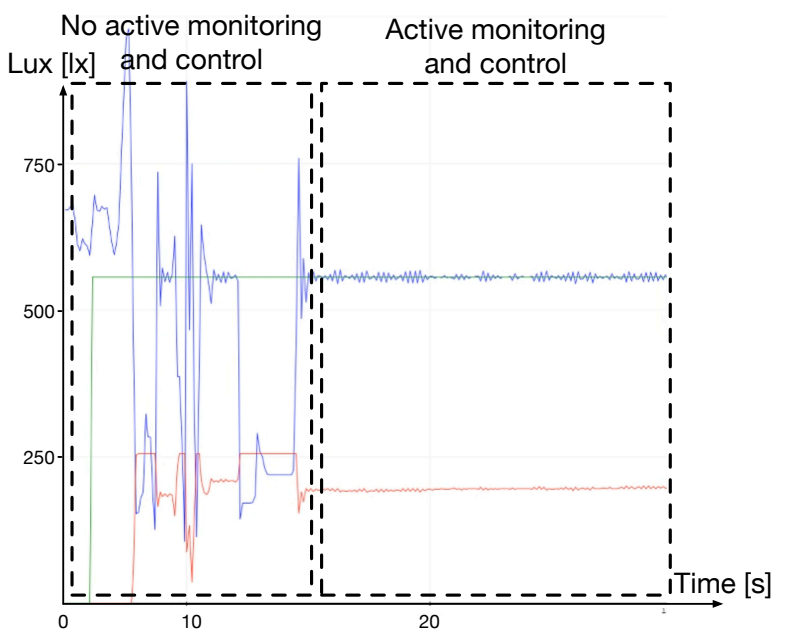

Figure 6. The collected sensor data can be monitored in real-time. A real-time plot for the control of the luminosity of one room. When active monitoring and control is applied, the measured luminosity closely tracks the desired threshold.

\section{Conclusion and future work}

This paper presented a new framework by leveraging the findings from earlier works related to ASD and by introducing novel additions to create a conducive workplace for people with ASD and similar challenges. Specifically, the designed framework uses the sensors of a smart-building in a cooperative manner and by combining them with personal sensors like the ones embedded on a smart-phone. The proposed system is further conceptually presented by implementing a prototyping mobile application along with a preliminary case study conducted on a physical scale model smart workplace. The Autismeforeningen i Norge (AN), which is the autism association in Norway, was involved during the development phase.

The system is built on open-source software and hardware and it can be used for testing different control algorithms as well as for training purposes. In the future, different control algorithms such as the ones discussed in $[17,18]$ may be tested.

Whilst the system is a preliminary prototype, a more immersive integration between portable and fixed sensor will be developed and tested in the future. A series of experiments will be further conducted and the gathered data will be analysed to validate the applicability in a real life scenario. A comprehensive experimental test will also make it possible to assess the proposed system from a human factor point of view and the findings will be vetted through experienced doctors. The 
needed improvements will be incorporated and tested in a continual manner. Special considerations will be given for ethical guidelines from medical perspectives by considering the need to have focus on information security to create confidence and trust for users. It will also be important to test the system with a variety of users to reflect the diversity of sensory challenges.

The future works will also include efforts towards a necessary standardisation process of the proposed framework to make it even more reliable for both the industrial and the academic practice. It is the opinion of the authors that the key to maximising the long-term, macroeconomic benefits relies on the closely integrated development of open content, open standards, and open source. The stable version of the proposed framework along with initial data (anonymised version) will be made available for non-profit research. The proposed system could have a broader use than ASD subjects. It could be useful in many situations also including healthy subjects or for people recovering from different pathologies or surgeries, e.g. eye surgery. These additional benefits may magnify both the social and economical benefits of the proposed system.

\section{Acknowledgement}

The authors gratefully acknowledge the contribution of Krystian Stanislaw Hoftun, Kim André Myre and Jarand Solberg Strømmen for the software implementation of the first prototype of the proposed system.

This work is supported by the Autismeforeningen i Norge (AN), which is the autism association in Norway. The current research consortium also include the leading Norwegian institute of technology NTNU - and a commercial entity - PRIMA AS 5, a Norwegian occupational health company provider, which delivers solutions to help companies and organisations with achieving more efficient well-being, and with implementing health promotion initiatives in the workplace. Another partner is Spesialistbedriften AS ${ }^{6}$, a company that employs people with Asperger's syndrome, providing them with a safe and evolving workplace. The consortium also amalgamates clinicians, professional occupational therapists and nurses in Norway, from St. Olav's University Hospital (SOHO), Norway. The consortium has applied for funding for a three-year research project (2019-2021) through the "ExtraStiftelsen Helse og Rehabilitering" funding scheme.

\footnotetext{
${ }^{5}$ http: //prima-as.no/

${ }^{6}$ http: //spesialistbedriften.no/
}

\section{References}

[1] C. Lord, E. Petkova, V. Hus, W. Gan, F. Lu, D. M. Martin, O. Ousley, L. Guy, R. Bernier, J. Gerdts, et al., "A multisite study of the clinical diagnosis of different autism spectrum disorders," Archives of general psychiatry, vol. 69, no. 3, pp. 306-313, 2012.

[2] F. de Ruiter, T. Micaelsen, T. K. Knutsen, R. Grung, I. Kjerkol, and K. Andersen, "Representantforslag 90 S," 2018.

[3] F. Tang, R. P. McMahan, and T. T. Allen, "Development of a low-cost tactile sleeve for autism intervention," in International symposium on Haptic, audio and visual environments and games (have), pp. 35-40, IEEE, 2014.

[4] M. J. Carley, Unemployed on the Autism Spectrum: How to Cope Productively with the Effects of Unemployment and Jobhunt with Confidence. Jessica Kingsley Publishers, 2016.

[5] D. S. Berger, Music therapy, sensory integration and the autistic child. Jessica Kingsley Publishers, 2002.

[6] R. Accordino, R. Comer, and W. B. Heller, "Searching for music's potential: A critical examination of research on music therapy with individuals with autism," Research in Autism Spectrum Disorders, vol. 1, no. 1, pp. 101-115, 2007.

[7] J. K. Kern, M. H. Trivedi, C. R. Garver, B. D. Grannemann, A. A. Andrews, J. S. Savla, D. G. Johnson, J. A. Mehta, and J. L. Schroeder, "The pattern of sensory processing abnormalities in autism," Autism, vol. 10, no. 5, pp. 480-494, 2006.

[8] A. E. Robertson and D. R. Simmons, "The sensory experiences of adults with autism spectrum disorder: A qualitative analysis," Perception, vol. 44, no. 5, pp. 569-586, 2015.

[9] L. Schor, P. Sommer, and R. Wattenhofer, "Towards a zero-configuration wireless sensor network architecture for smart buildings," in Proceedings of the First ACM Workshop on Embedded Sensing Systems for Energy-Efficiency in Buildings, pp. 31-36, ACM, 2009.

[10] G. P. Hancke, G. P. Hancke Jr, et al., "The role of advanced sensing in smart cities," Sensors, vol. 13, no. 1, pp. 393-425, 2012.

[11] J. Rumbaugh, I. Jacobson, and G. Booch, Unified modeling language reference manual, the. Pearson Higher Education, 2004. 
[12] M. A. E.-L. Mowad, A. Fathy, and A. Hafez, "Smart home automated control system using android application and microcontroller," International Journal of Scientific \& Engineering Research, vol. 5, no. 5, pp. 935-939, 2014.

[13] I. Pan, S. Das, and A. Gupta, "Tuning of an optimal fuzzy pid controller with stochastic algorithms for networked control systems with random time delay," ISA transactions, vol. 50, no. 1, pp. 28-36, 2011.

[14] A. Developers, "What is android," 2011.

[15] Arduino, "Arduino, an open-source electronics prototyping platform," June 2018.

[16] R. Piyare and M. Tazil, "Bluetooth based home automation system using cell phone," in Proc. of the 15th International Symposium on Consumer Electronics (ISCE), pp. 192-195, IEEE, 2011.

[17] F. Sanfilippo and C. Pacchierotti, "A wearable haptic system for the health monitoring of elderly people in smart cities," International Journal of Online Engineering (iJOE), 2018. Manuscript accepted for publication.

[18] F. Sanfilippo and K. Pettersen, "A sensor fusion wearable health-monitoring system with haptic feedback," in Proc. of the 11th IEEE International Conference on Innovations in Information Technology (IIT'15), Dubai, United Arab Emirates, pp. 241-245, 2015. 